\title{
Aberrant expression of miRNAs in epilepsy
}

\author{
Soudeh Ghafouri-Fard ${ }^{1} \cdot$ Bashdar Mahmud Hussen $^{2,3} \cdot$ Atefe Abak $^{4} \cdot$ Mohammad Taheri $^{5,6} \cdot$ Reza Jalili Khoshnoud $^{7}$
}

Received: 9 November 2021 / Accepted: 21 January 2022 / Published online: 28 January 2022

(c) The Author(s) 2022

\begin{abstract}
Epilepsy is manifested by intermittent convulsions and alterations in consciousness. This disorder has serious effects on daily functions and physical and mental health of affected patients. A variety of temporary irregularities in the function of brain can results in epilepsy. The molecular mechanism of epilepsy and the underlying causes of abnormal apoptotic responses in neurons, dysregulation of regenerative mechanisms in glial cells and abnormal immune reactions in the context of epilepsy are not clear. microRNAs (miRNAs) as important regulators of cell apoptosis as well as regenerative and immune responses have been shown to affect pathologic events in epilepsy. In the current review, we aimed at defining the role of miRNAs in the pathophysiology of epilepsy. We have listed dysregulated miRNAs in animal models of epilepsy and human subjects. miR-25-3p, miR-494, miR-139-5p, miR-101a-3p, miR-344a, miR-129, miR-298 and miR-187 are among downregulated miRNAs in epilepsy. Moreover, expressions of miR-132, miR-146a, miR-181a and miR-155 have been reported to be increased in epilepsy. A number of genetic variants within miRNAs can affect risk of epilepsy. We discuss the role of miRNAs in the development of epilepsy.
\end{abstract}

Keywords miRNA $\cdot$ Epilepsy $\cdot$ Biomarker $\cdot$ Expression $\cdot$ Diagnosis

Mohammad Taheri

mohammad.taheri@uni-jena.de

Reza Jalili Khoshnoud

drkhoshnoud@gmail.com

1 Department of Medical Genetics, School of Medicine, Shahid Beheshti University of Medical Sciences, Tehran, Iran

2 Department of Pharmacognosy, College of Pharmacy, Hawler Medical University, Erbil, Kurdistan Region, Iraq

3 Center of Research and Strategic Studies, Lebanese French University, Erbil, Kurdistan Region, Iraq

4 Men's Health and Reproductive Health Research Center, Shahid Beheshti University of Medical Sciences, Tehran, Iran

5 Institute of Human Genetics, Jena University Hospital, Jena, Germany

6 Skull Base Research Center, Loghman Hakim Hospital, Shahid Beheshti University of Medical Sciences, Tehran, Iran

7 Functional Neurosurgery Research Center, Shahid Beheshti University of Medical Sciences, Tehran, Iran

\section{Introduction}

Epilepsy is resulted from a variety of temporary irregularities in the function of brain due to an anomalous, extremely synchronous discharge of neuronal cells. Clinically, it is manifested by intermittent convulsions and alterations in consciousness, which has serious effects on daily functions and physical and mental health of affected patients [1]. Alterations in various gene patterns in the neurons can lead to the abnormal protein metabolism detected in the neurons of patients with this disorder [2,3]. The molecular mechanism of epilepsy and the underlying causes of abnormal apoptotic responses in neurons, dysregulation of regenerative mechanisms in glial cells and abnormal immune reactions need to be clarified. microRNAs (miRNAs) as important regulators of cell apoptosis as well as regenerative and immune responses [4], are putative contributors in the pathogenesis of epilepsy. These small noncoding RNAs are approximately 19-22 nucleotides long and regulate gene expression through silencing mechanisms at posttranscriptional level [4]. It is estimated that more than one third of the human genome is under influence of regulatory roles of miRNAs [4]. Notably, nearly all regulatory mechanisms of expression of genes such as transcription factors as well as 
epigenetic factors have been found to act irregularly in the course of epilepsy [5]. As miRNAs can affect expression of transcription factors, dysregulation of miRNAs can influence epilepsy from different direct and indirect routes. In the current paper, we discuss the impact of dysregulation of miRNAs on development of epilepsy. We have searched PubMed and Google Scholar databases with key words "microRNA" or "miRNA" AND "epilepsy" or "seizure". After assessment of Abstracts and full texts of retrieved articles, we have included all relevant original papers in animal models and human subjects.

\section{Down-regulated miRNAs in epilepsy}

\section{Refractory epilepsy}

miR-139-5p is another miRNA with possible protective role against epilepsy. Expression of this miRNA has been found to be reduced in the sera of children with refractory epilepsy, parallel with up-regulation of expression of multidrug resistance-associated protein 1 (MRP1). The same expression pattern has been detected in the brain samples of rat models of refractory epilepsy. Functional studies have confirmed that MRP1 is targeted by miR-139-5p. Transfection of plasmids into the hippocampus of drug-resistant rats has verified the effects of miR-139-5p up-regulation or MRP1 silencing in reduction of neuron apoptosis, enhancement of neuron survival, and amendment of neuron injury. Thus, miR-139-5p/ MRP1 axis can reduce resistance of refractory epilepsy to antiepileptic medications [6]. Expression of miR-34c-5p has also been reported to be decreased in patients with refractory epilepsy compared to controls. This miRNA targets the inflammation-related mediator gene HMGB1. Experiments in rat models of kainic acid (KA)-induced epilepsy have shown down-regulation of miR-34c-5p and up-regulation of HMGB 1 and IL-1 $\beta$ in drug-resistant epileptic animals compared to drug-sensitive epileptic animals. Moreover, hippocampal neuron loss has been more prominent in drugresistant epileptic animals. Thus, down-regulation of miR$34 c-5 p$ in refractory epilepsy aggravates neuroinflammatory responses, which exacerbates hippocampal neuron loss. These findings indicate that miR-34c-5p might be a possible noninvasive marker for refractory epilepsy [7]. miR-153 is another miRNA whose dysregulation is implicated in the pathogenesis of refractory epilepsy. This miRNA possibly acts through regulation of HIF- $1 \alpha$ expression (8).

\section{Other types of epilepsy}

$\mathrm{Li}$ et al. have examined expression levels of miR-15a-5p in serum samples of children with temporal lobe epilepsy (TLE). They have also cultured primary hippocampal cells in magnesium-deficient condition to simulate TLE. Their experiments have demonstrated down-regulation of miR$15 a-5 p$ in sera of children with TLE. Notably, miR-15a-5p has been confirmed to be an appropriate marker with proper specificity and specificity values for diagnosis of TLE in children. Moreover, magnesium-deficient condition has reduced expression levels of miR-15a-5p in hippocampal cells. On the other hand, up-regulation of miR-15a-5p has ameliorated TLE-associated decrease in cell viability, and attenuated the TLE-induced apoptosis. Therefore, miR$15 a-5 p$ has been suggested a promising marker for the detection of TLE in children [9].

Another experiment in a rat model of epilepsy has shown that miR-21-5p can bind to STAT3. Expressions of caspase- 3 and Bax have been higher, while expression of $\mathrm{Bcl}-2$ has been lower in animals that received miR-21-5p inhibitor. miR-21-5p inhibitor has also resulted in loss of hippocampal neurons and induction of apoptosis in these cells, while suppression of STAT3 expression has led to opposite effects. Moreover, IL-6 levels have been higher in those received miR-21-5p inhibitor. Therefore, miR-21-5p is able to suppress expression of STAT3, decrease IL-6 levels and reduce loss of hippocampal neurons, thus protecting hippocampal neurons from deteriorating effects of epilepsy [10]. Table 1 shows the list of down-regulated miRNAs in epilepsy. Figure 1 illustrates the role of several miRNAs in epilepsy through regulating the NF- $\mathrm{KB}$ signaling pathway.

\section{Up-regulated miRNAs in epilepsy}

Another experiment in epileptic rats has shown elevation of miR-103a and GFAP levels, higher quantity of apoptotic neurons, down-regulation of BDNF and reduction in the numbers of surviving neurons in hippocampal tissues of epileptic rats. Suppression of miR-103a has led to downregulation of GFAP, up-regulation of BDNF and reduction in the number of apoptotic neurons, while enhancing the proportion of surviving neurons. Therefore, suppression of miR-103a results in the activation of astrocytes in hippocampus and amends neuronal damage in epileptic rats through regulation of expression of BDNF [23]. In addition, miR-27a-3p has been shown to be over-expressed in the hippocampal cells of epileptic rats and in KA-treated neurons. Notably, miR-27a-3p silencing has relieved epileptic seizures in animal models. In addition, miR-27a-3p silencing has suppressed apoptosis of hippocampal neurons in rat models of epilepsy, increased expression of $\mathrm{Bcl} 2$, and reduced levels of Bax and Caspase3. Moreover, miR-27a-3p silencing has efficiently decreased expressions of IL-1B, IL-6, and TNF- $\alpha$ in hippocampal neurons. These effects are mediated through modulation of expression of MAP2K4, since this gene is a direct target of miR-27a-3p. miR-27a-3p silencing has also enhanced survival of KA-treated neurons, 


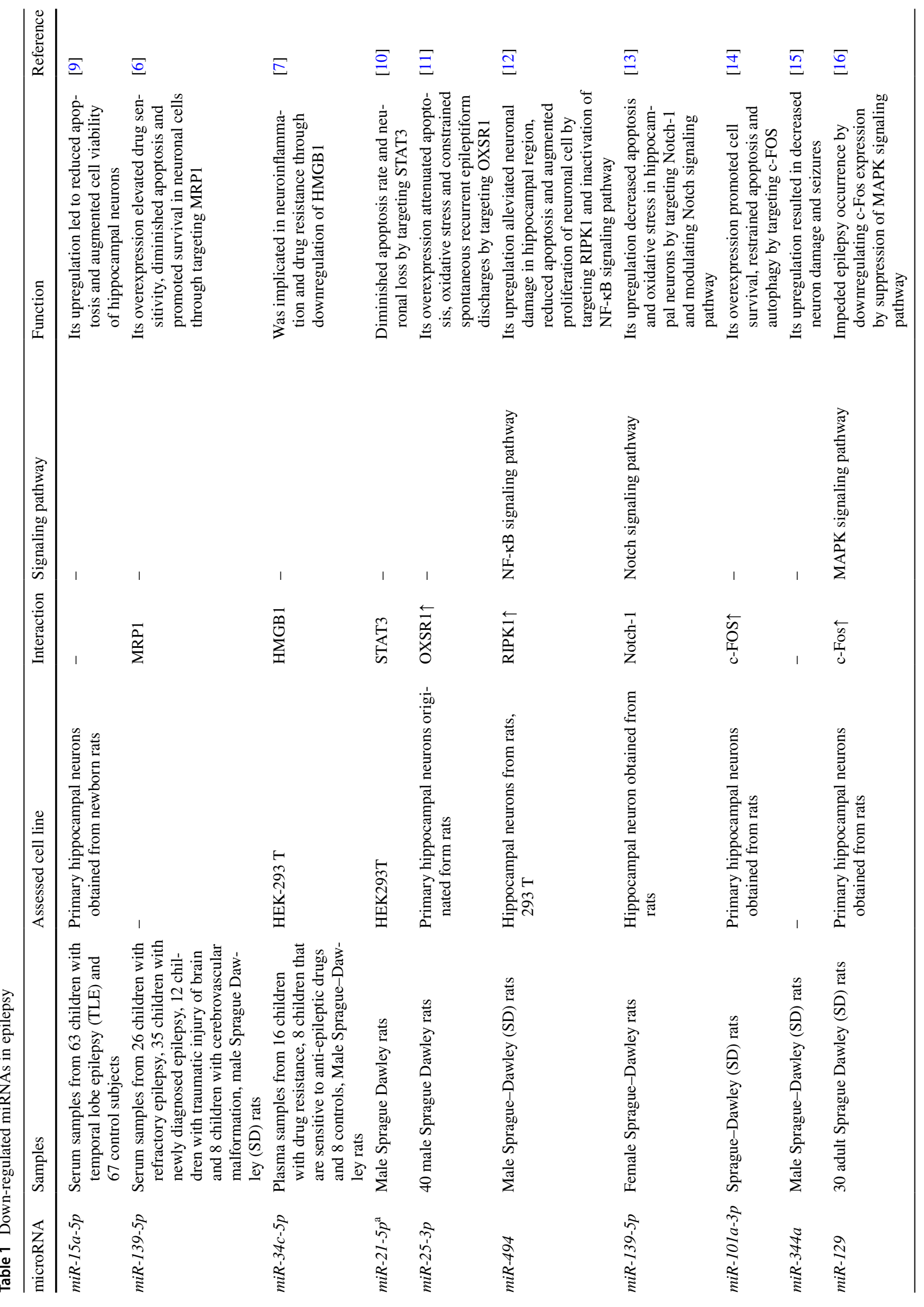




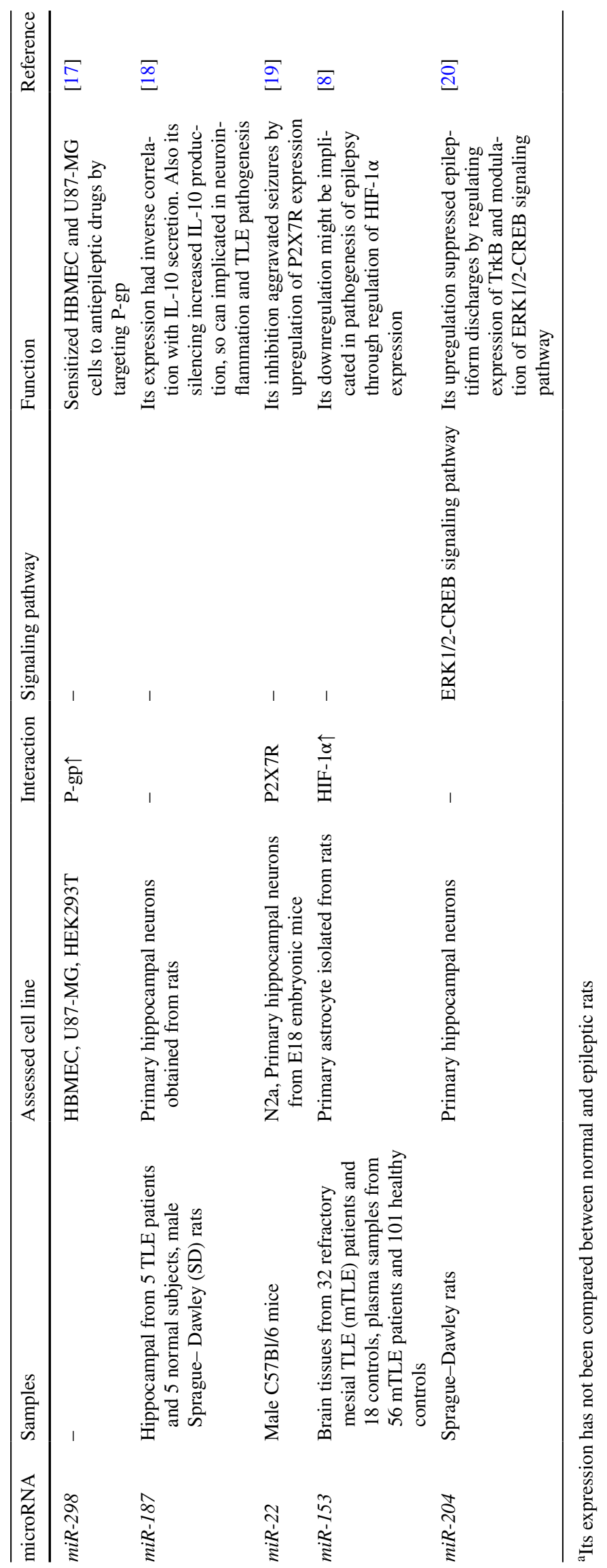




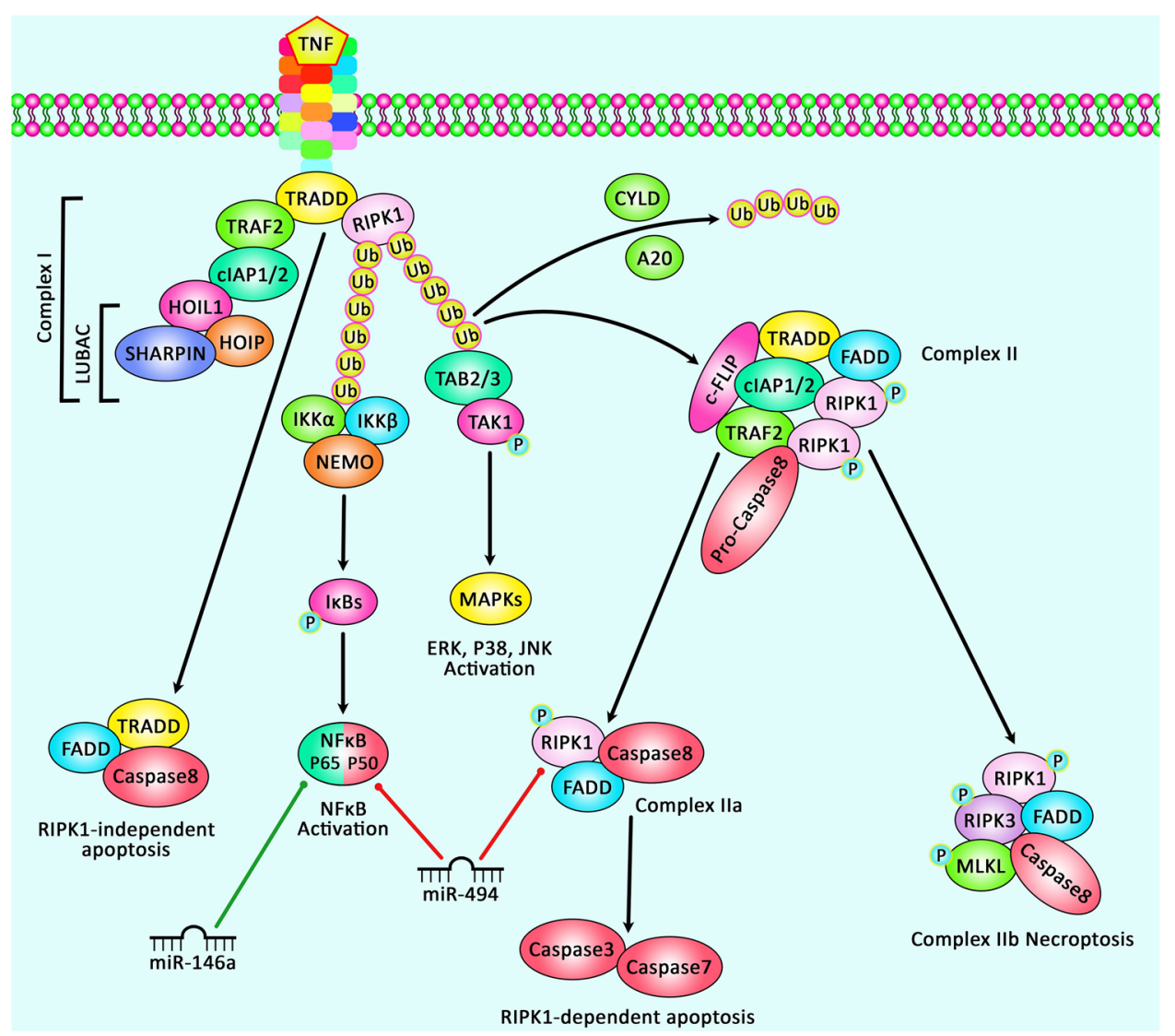

Fig. 1 A schematic representation of the role of various miRNAs in modulating the NF- $\kappa \mathrm{B}$ signaling cascade in epilepsy. After the binding of TNF to its receptor and stimulating it, TNFR1 could undergo a conformational alteration and recruit various proteins to create complex I, including TRADD, TRAF2/5, RIPK1, cIAP1/2, SHARPIN, HOIL-1, and HOIP. In complex I, the K63-linked ubiquitination of RIPK1 via cIAP1/2, leading to the binding of TAB2/3 and NEMO, modulating the recruitment of the TAK $1 / \mathrm{TAB}$ complex and the IKK $\alpha / \mathrm{IKK} \beta / \mathrm{NEMO}$ complex that could result in inducing the NF- $\mathrm{KB}$ cascade and cell survival. Eventually, the ubiquitinated RIPK1 could trigger the ubiquitination and proteasomal degradation of $\mathrm{IkB}$, leading to nuclear transition of the released NF-kB and its upregulation. Besides, RIPK1 could regulate the overexpression of MAPKs, ERK, P38 and JNK that are induced via TNF- $\alpha$. Destabilization of complex I leads to the creation of complex IIa, that includes TRADD, FADD and caspase-8. Furthermore, complex IIa contains the adaptor FADD,

suppressed their apoptosis, increased expression of Bcl2 , and reduced expressions of Bax and Caspase 3 through modulation of MAP2K4. Therefore, miR-27a-3p silencing protects against epilepsy-associated inflammatory responses and apoptosis of hippocampal neurons through influencing expression of MAP2K4 [24]. miR-132 is one of the most frequently upregulated miRNAs in animal models of TLE. This miRNA can affect functions of both neurons [25] and glial cells [26]. Expression of miR-132 has been found to be elevated in the hippocampal cells of human and rat epileptic subjects, principally in glial cells. Ectopic expression of miR-132 in human primary astrocytes has led to reduction caspase 8, and RIPK1, and regulates the upregulation of caspase 8, then caspase 3, and caspase 7, resulting in apoptosis. When caspase 8 is suppressed, RIPK1 could merge with RHIM in RIPK3 to create complex IIb, creating RIPK3 oligomerization and autophosphorylation. Then, RIP3 could play an effective role in recruiting and phosphorylating MLKL, resulting in the necrosome [21, 22]. Growing evidences confirm that aberrant expression of miRNAs could have a crucial role in epilepsy. As an illustration, recent study has detected that upregulation of miR-494 could downregulate the expression level of RIPK1, triggering a suppression of the NF- $\mathrm{KB}$ signaling cascade and promotion of cell proliferation, and inhibition of apoptosis of hippocampal neurons in epilepsy, therefore attenuating the neuron injury and epilepsy development [12]. Furthermore, another research has illustrated that NF- $\kappa$ B could be modulated via miR-146a which has an important role in the pathogenesis of epilepsy development [16]

of expression of a number of pro-epileptogenic genes, namely COX-2, IL-1 $\beta$, TGF- $\beta 2$, CCL2, and MMP3 [26]. The interaction between miR-132 and p250GAP/Cdc42 axis has been found as the uderlying mechanism of contribution of this miRNA in the epileptogenesis, based on the experiments performed in the hippocampal neurons cultures [25]. Another study has revealed a significant elevation in the levels of miR-132 and BDNF transcripts in the hippocampal neurons culture model of status epilepticus produced by $\mathrm{Mg}(2+)$-deficient medium. Activation of TrkB.FL by pretreatment with BDNF has partially suppressed the $\mathrm{Mg}(2+)$ free associated unremitting high-frequency epileptiform 
discharges, whereas up-regulation of miR-132 has aggravated epileptiform discharges. miR-132 has also been found to partake in the postepileptic augmentation of high voltage dependent calcium channels. Therefore, miR-132 has proepileptic effects via modulating BDNF/TrkB pathway in the hippocampal neuron culture model of status epilepticus [27]. miR-146a is another up-regulated miRNA in the course of epilepsy. Experiments in a rat model of TLE have shown up-regulation of miR-146a in the hippocampal tissues. miR146a knock down has remarkably amended neuron injury and cell apoptosis in rat hippocampus, reduced MDA, IL-1 $\beta$, IL-6, and IL-18 expressions and enhanced SOD levels in this tissue. Moreover, miR-146a silencing has reduced expressions of caspase-9, GFAP, Notch-1, and Hes-1 in the hippocampus of animal models of TLE. Functional studies have shown Notch-1 as the target of miR-146a. Thus, miR146a silencing alleviates neuron injury in the hippocampus of animal models of TLE through inhibiting expression of Notch-1 [28]. Another study has demonstrated high levels of miR-146a in the lithium-pilocarpine- induced model of epilepsy. miR-146 silencing has led to reduction of IL-1 $\beta$, IL- 6 and TNF- $\alpha$ levels. Moreover, expressions of P-gp and p-P65/P65 have been decreased after miR-146a silencing, while expressions of $\mathrm{Bcl}-2 / \mathrm{Bax}$ have been increased following this intervention [16]. miR-181a is another upregulated miRNA in epilepsy. Its inhibition has resulted in protective effects against epilepsy, reduced apoptosis and decreased activation of microglia and astrocyte by upregulating SIRT1 [29]. Moreover, its silencing has constrained apoptosis in hippocampal neurons [30]. Expression levels of miR-21-5p and mTOR have been shown to be increased in rats during acute, latent, and chronic phases of epilepsy parallel with down-regulation of PTEN. In vivo suppression of miR-21-5p has led to down-regulation of mTOR and upregulation of PTEN. miR-21-5p silencing has also reduced the quantity of abnormal spikes in EEG and diminished the neuron defects. Moreover, this intervention has ameliorated epilepsy-induced cognitive and memory damages in vivo. Targeting PTEN-mTOR axis by miR-21-5p has been identified as the molecular mechanism of participation of this miRNA in the pathogenesis of epilepsy [31]. Table 2 shows up-regulated miRNAs in epilepsy. Figure 2 represents the role of various miRNAs via regulating the Notch signaling cascade in epilepsy.

\section{Diagnostic/prognostic role of miRNAs in epilepsy}

Several miRNAs have been found to have potential applicability as diagnostic or prognostic markers in epilepsy. For instance, miR-15a-5p has diagnostic power of 0.908 with $82.5 \%$ sensitivity and $88.1 \%$ specificity in diagnosis of TLE children from healthy matched controls [9]. Expression of hsa-miR-134 has been found to be deceased in patients with mesial TLE (MTLE) but not in patients with focal cortical dysplasia (FCD) when compared to healthy subjects. hsamiR-134 could separate MTLE patients from controls with diagnostic power of 0.75 . This result has been validated an independent cohort of patients with MTLE including both refractory and drug-responsive patients. Therefore, hsamiR-134 has been suggested as a marker for MTLE in an independent manner from their response antiepileptic drugs or existence of MRI signs of hippocampal sclerosis [57]. Another study has investigated the role of miR-27a-3p, miR$328-3 p$ and miR-654-3p as putative circulating biomarkers for epilepsy diagnosis and prediction of outcome of surgical intervention in a cohort of MTLE with hippocampal sclerosis (MTLE-HS) including those with good surgical prognosis (Engel I) and those with unfavorable surgical prognosis (Engel III-IV). miR-27a-3p has not been validated as a circulatory marker for diagnostic or prognostic purposes. However, miR-328-3p could differentiate controls from Engel I, controls from Engel III-IV and controls from Engel I+ Engel III-IV patients with diagnostic power values of $90.3 \%, 96.8 \%$ and $93.5 \%$, respectively. In addition, miR654-3p could differentiate controls from Engel I patients as well as patients with unfavorable from favorable surgical outcome with lower values [58].

Although the diagnostic power of several miRNAs has been assessed in epilepsy, a major drawback of the majority of studies is lack of confirmation of the obtained results in independent cohorts of patients. Moreover, only few miRNAs have been assessed in more than one study. For instance, miR-134 could differentiate patients with MTLE from controls with diagnostic power of 0.75 [57]. It could also predict development of drug-resistance with power of 0.61 [59]. Since studies in this regard are not ample, it is not possible to find the impact of sample size, variations in the methodology, or geographic origin of the cohorts studied on the obtained results.

Table 3 shows the diagnostic and prognostic role of miRNAs in epilepsy.

\section{miRNAs polymorphisms in epilepsy}

Association between single nucleotide polymorphisms within miR-146a and risk of epilepsy has been appraised in Brazilian, Chinese and Italian patients (Table 4). GC genotype of rs2910164 has been associated with higher risk of drug-resistant epilepsy among Brazilians. In addition, GC and CC genotypes of this SNP has been associated with low expression of miR-146a in epileptogenic tissues compared to GG genotype [67]. rs57095329 within this gene has also been correlated with risk of drug resistant epilepsy among Chinese patients [68]. In addition, A allele of rs57095329 has been associated with decreased frequency of seizures in drug resistant epilepsy patients [68]. However, rs2910164 


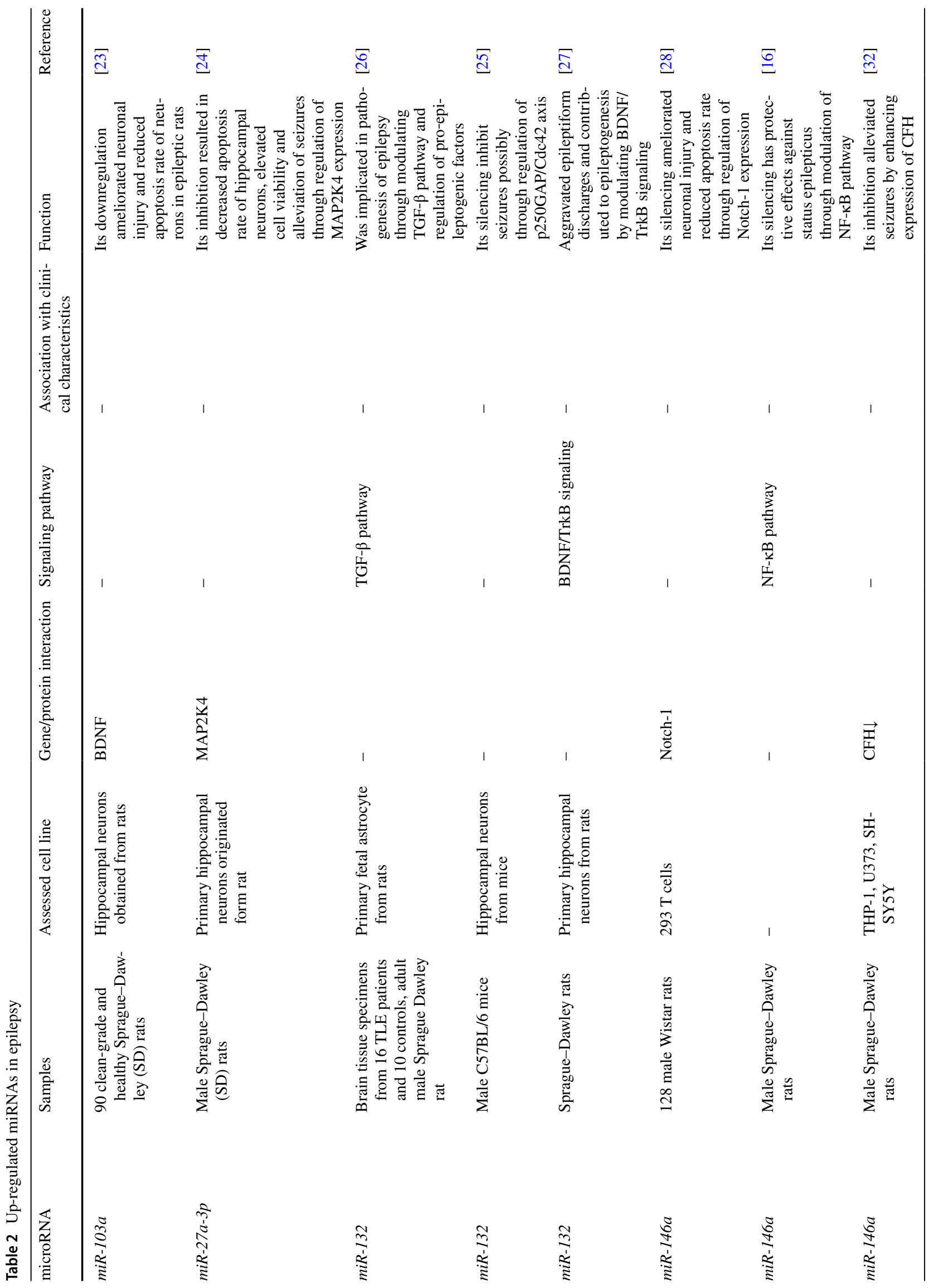




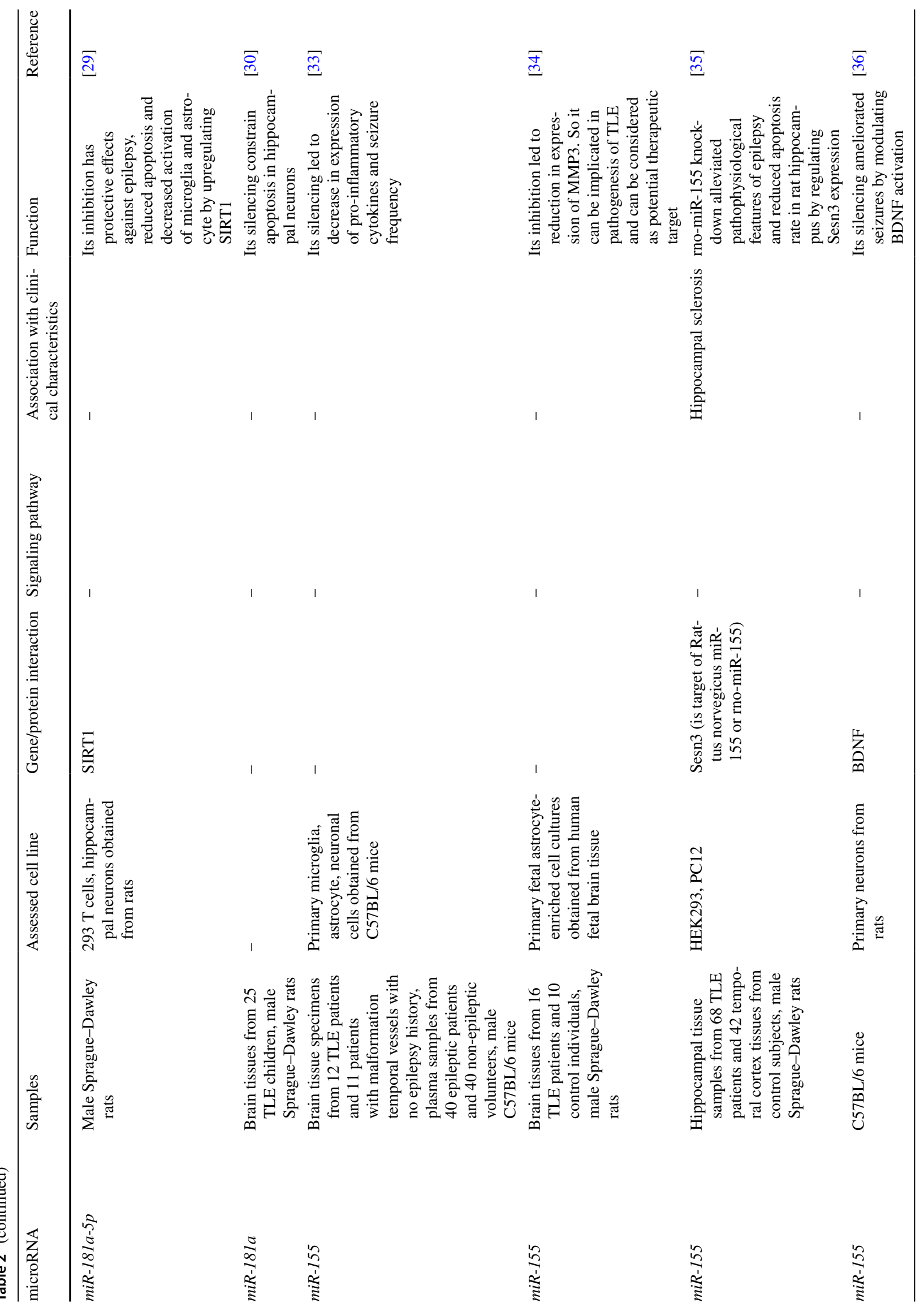




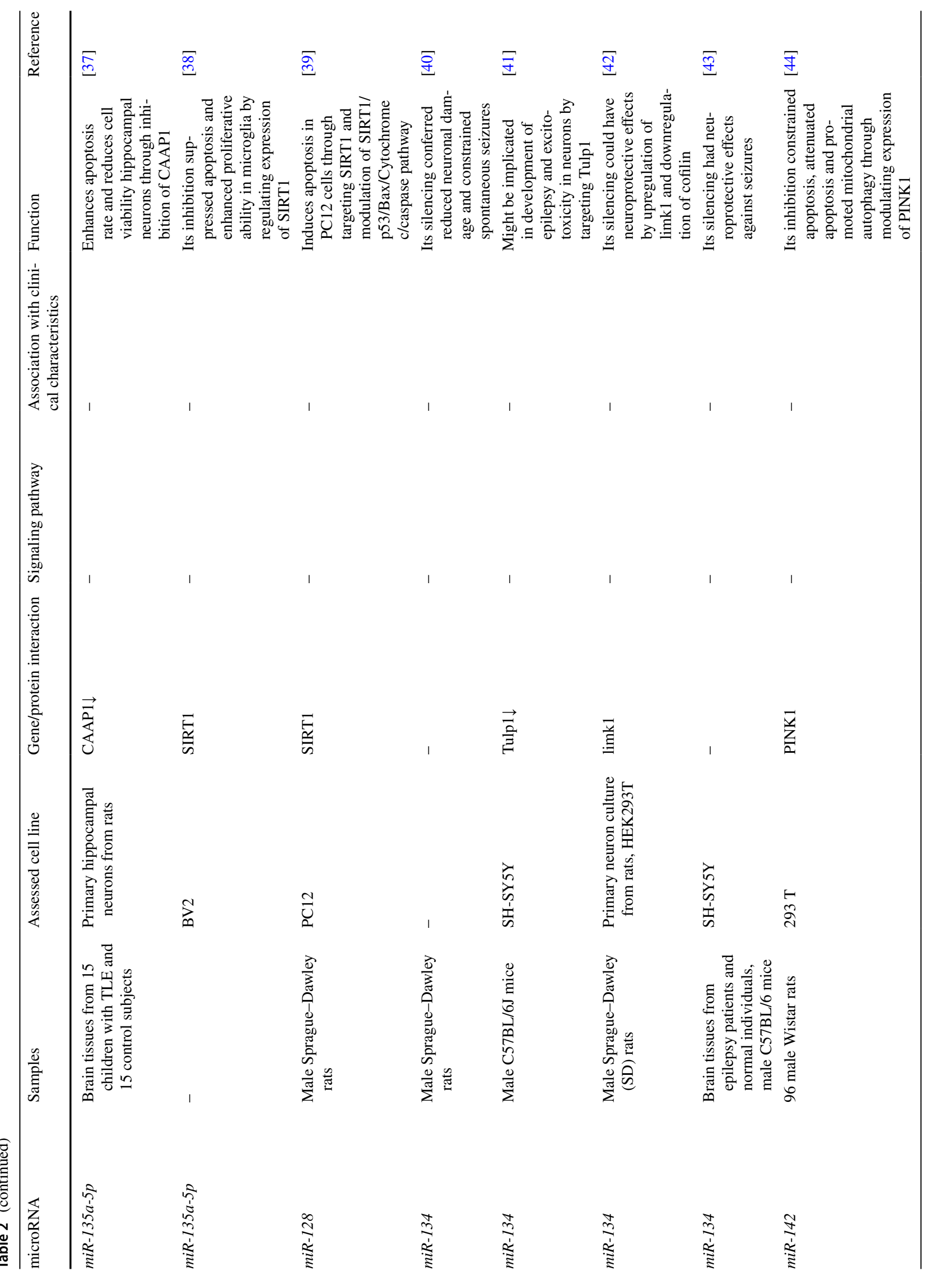




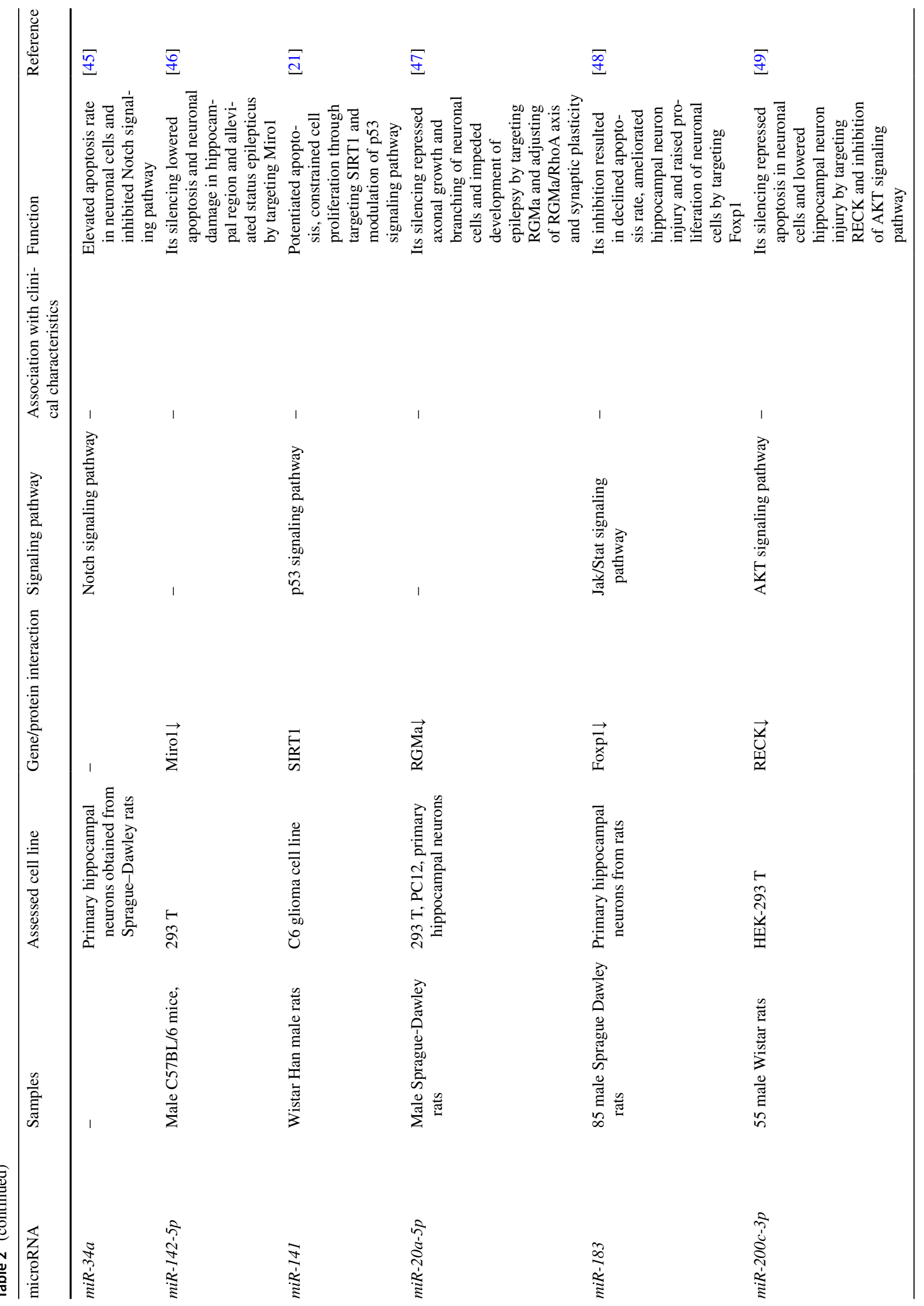




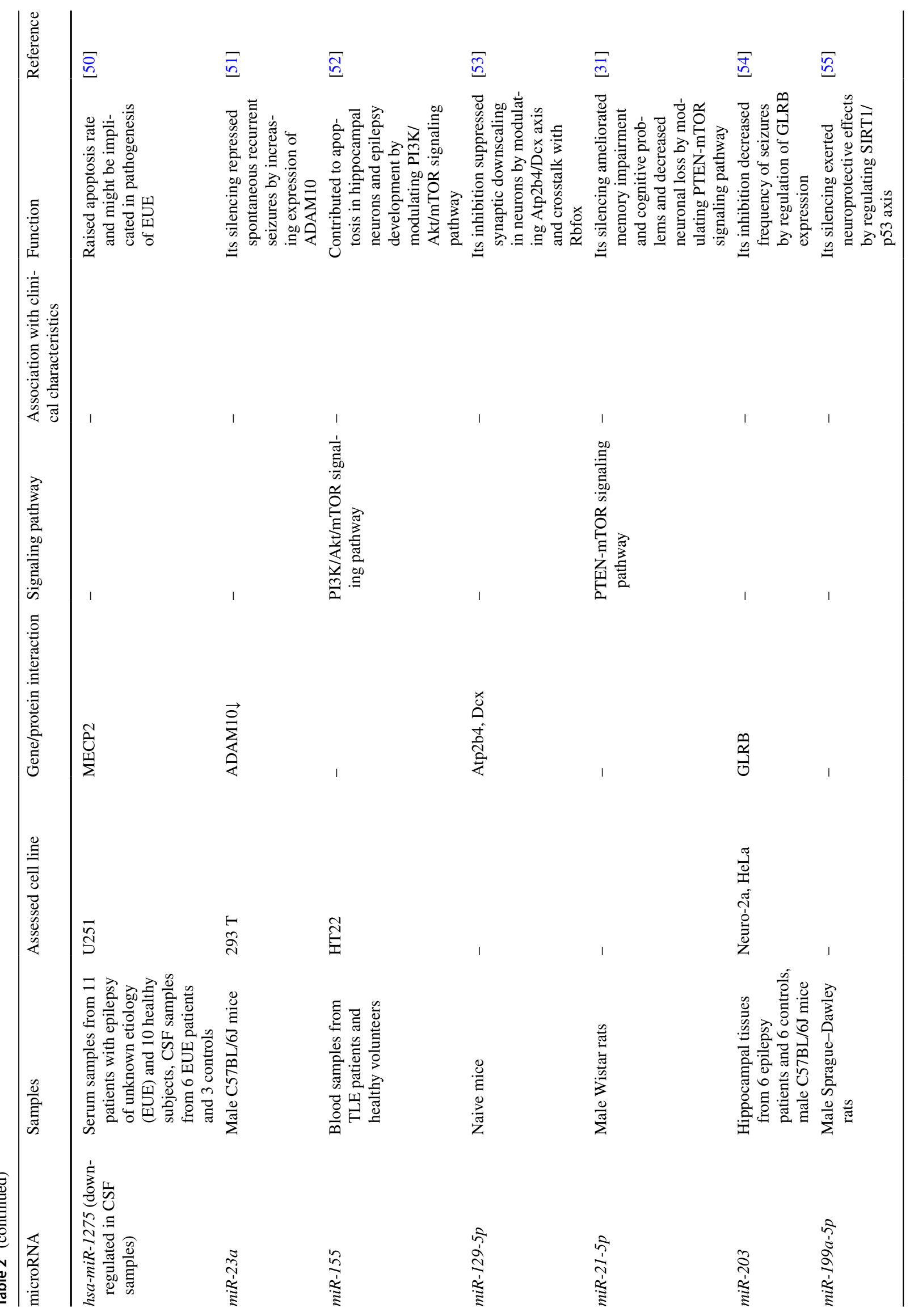




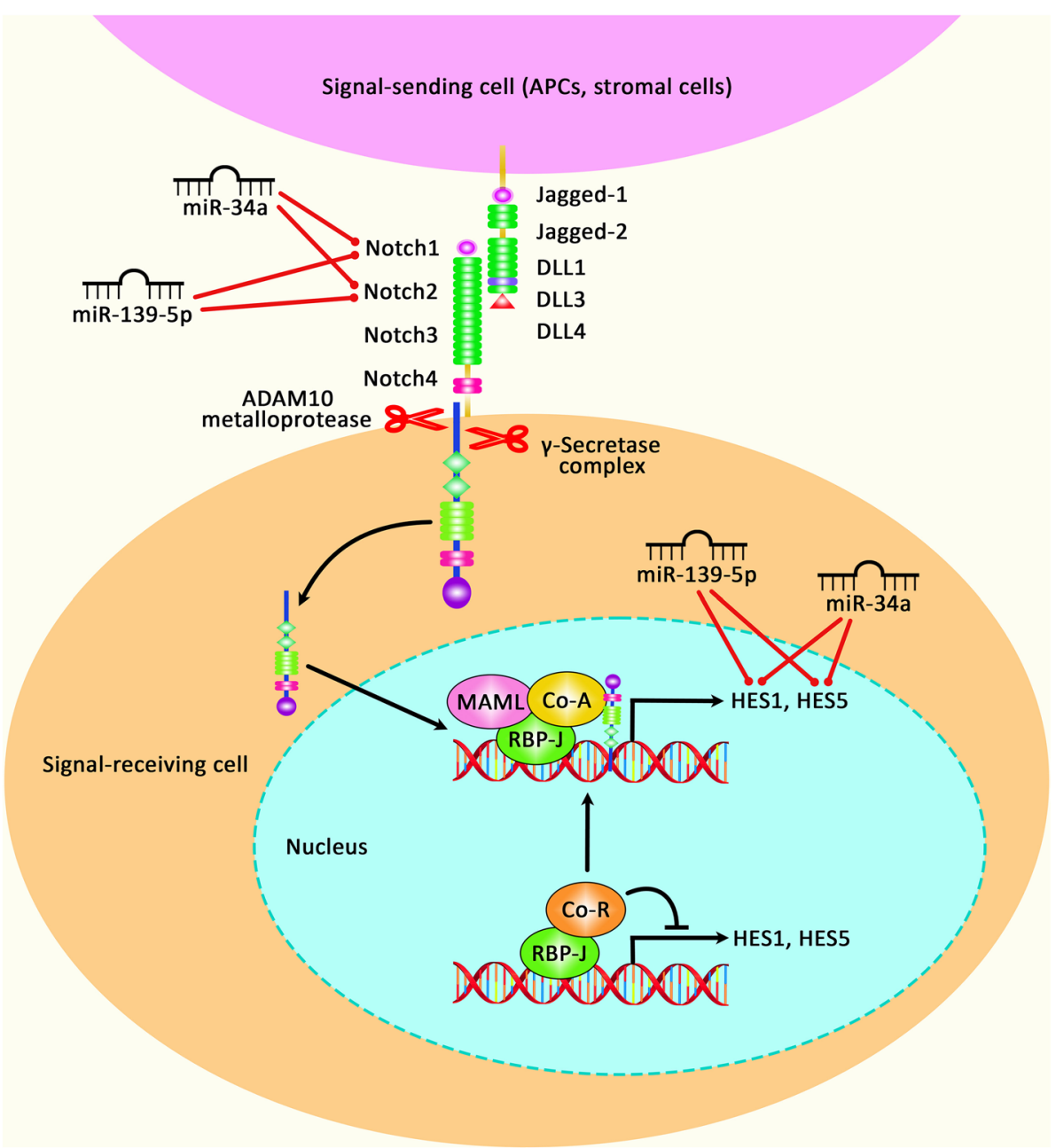

Fig. 2 A schematic diagram of the role of several miRNAs in regulating the Notch signaling pathway in epilepsy. Interaction between Notch and Delta or Jagged that exist on the surface of signal-sending cells could modulate the cleavage of Notch protein via ADAM10 metalloproteases and $\gamma$-secretase to create NICD. NICD could transfer to the nucleus to create a heterotrimer with RBP-J and MAML to promote transcription of various target genes, including HES1 and HES5 [56]. Mounting evidence indicates that overexpression of various miRNAs could have a significant role in triggering epilepsy through Notch signaling cascade. Recent research has represented

has not been associated with risk of TLE among Italians [69].

\section{Discussion}

miRNAs have been shown to affect several aspects of epliptogenesis. Modulation of apoptosis and survival of neurons and regulation of inflammatory responses are the most appreciated mechanisms of involvement of miRNAs in the pathogenesis of epilepsy. In addition to direct effects of miRNAs on molecular pathways in neurons, they can that downexpression of miRNA-34a could have a crucial role in suppressing epileptiform discharges via modulating Notch signaling and apoptosis in the rat hippocampal neuronal model of SREDs. In fact, SREDs could Induce the promotion of miR-34a expression level and decrease of the expression of Notch signaling (including Notch1, Notch2, Hes1 and Hes5) [45]. In addition, another study has demonstrated that overexpression of miR-139-5p could suppress spontaneous recurrent epileptiform discharge-induced oxidative stress and apoptosis through modulating the Notch pathway [13]

affect functions of reactive glial cells which potentially regulate inflammatory responses in the brain and remodeling of the extracellular matrix [26]. miR-132 and miR$146 \mathrm{a}$ are among the mostly assessed miRNAs in the animal models of epilepsy. These miRNAs have been found to affect several targets and pathways during epileptogenesis. For instance, miR-132 has interactions with TGF- $\beta$ and BDNF/TrkB signaling pathways in glial cells and neurons, respectively. miR-146a affects activity of Notch and $\mathrm{NF}-\kappa \mathrm{B}$ pathways in this context. SIRT1 and BDNF have been identified as molecular targets of several miRNAs in the context of epilepsy. 


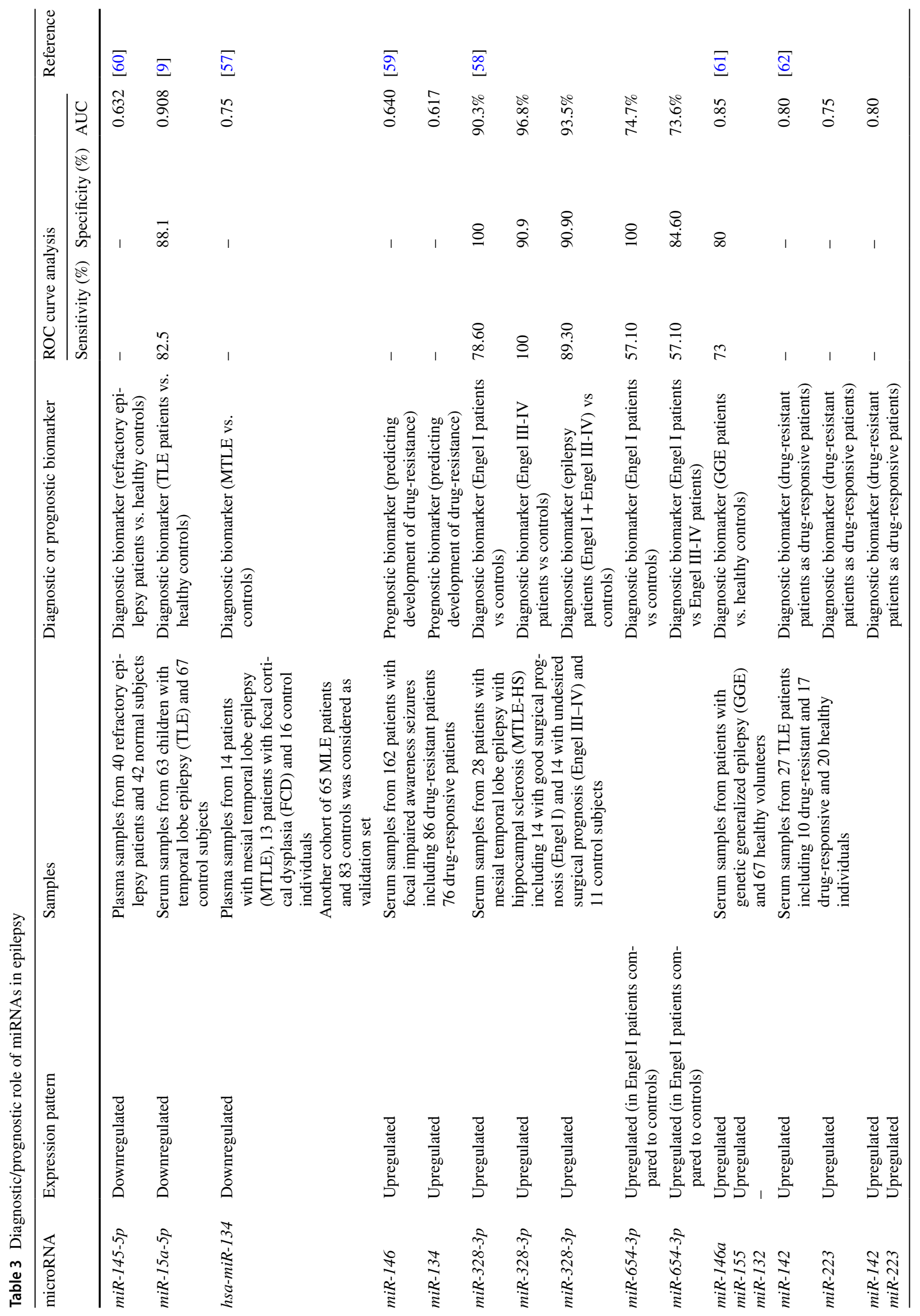




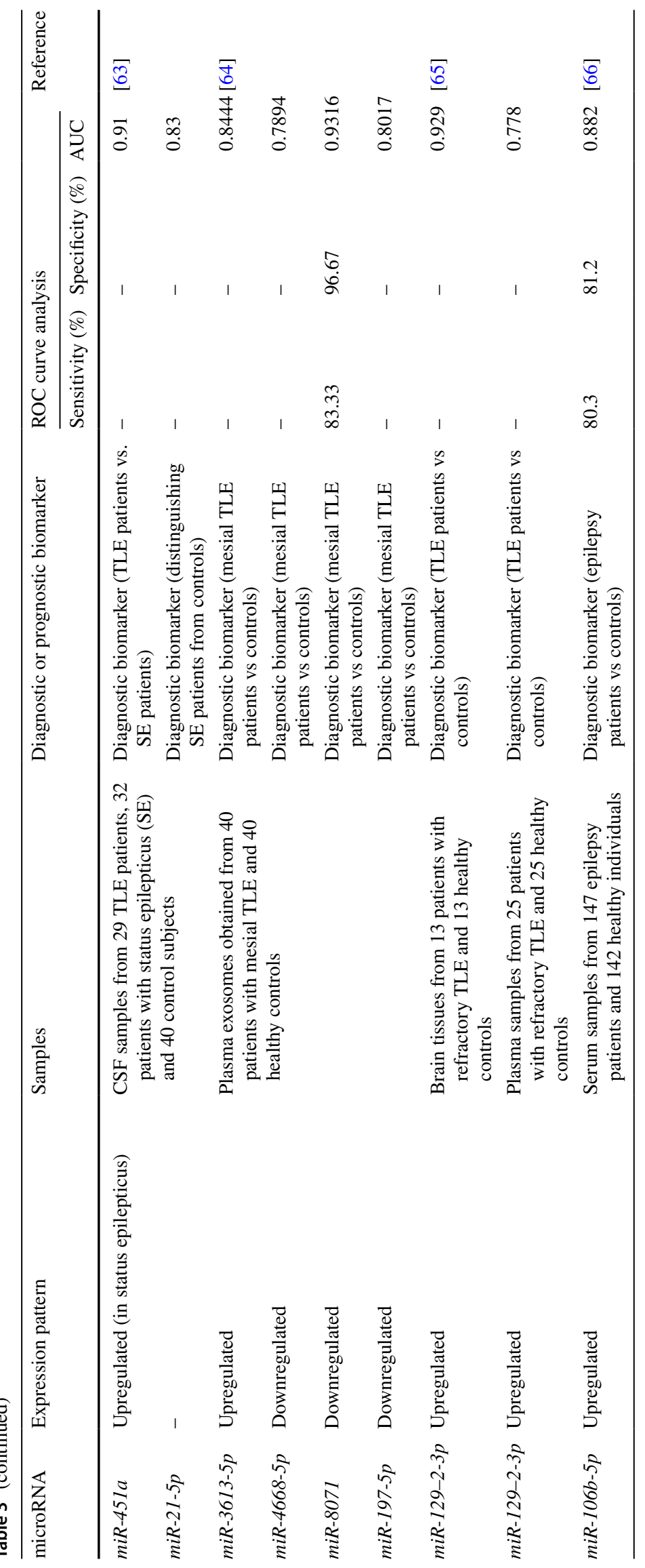


miRNAs have also diagnostic and prognostic functions in epilepsy. Some miRNAs such as miR-15a-5p, miR-328-3p, miR-129-2-3p and miR-106b-5p have been suggested as appropriate diagnostic markers in epilepsy, while miR-146 and miR-134 has been proposed as prognostic markers with mediocre performance. Moreover, miRNAs can modulate response of patients with refractory epilepsy to antiepileptic medications [6]. Therefore, miRNA-modulating therapeutic options might be used as alternative therapies for enhancing efficacy of antiepileptic drugs. Moreover, animal studies have shown that miRNA-targeting modalities might amend epilepsy-induced cognitive and behavioral impairments.

As the effects of miRNAs on glial cells and neurons might be exerted through different routes and even in different directions, miRNA-modulating therapies should be assessed in different cell types to validate their beneficial effects in each cell types.

\section{Conclusion}

In brief, several miRNAs have been shown to be dysregulated in brain tissues and serum samples of patients with epilepsy and different animal models of this neurological condition. Abnormal levels of these miRNAs in the serum samples show their potential as biomarkers for prediction of epilepsy. However, the results of these studies should be verified in independent cohorts from different stages of epilepsy. Contribution of genetic variants within miRNA coding genes in risk of epilepsy or resistance to antiepileptic drugs is another research area which should be explored in future.

Author contributions SGF wrote the manuscript and revised it. MT designed and supervised the study. BMH, AF and MS collected the data and designed the tables and figures. All authors read and approved the submitted manuscript.

Funding Open Access funding enabled and organized by Projekt DEAL.

Data availability Data sharing not applicable to this article as no datasets were generated or analysed during the current study.

\section{Declarations}

Conflict of interest The authors declare they have no conflict of interest.

Open Access This article is licensed under a Creative Commons Attribution 4.0 International License, which permits use, sharing, adaptation, distribution and reproduction in any medium or format, as long as you give appropriate credit to the original author(s) and the source, provide a link to the Creative Commons licence, and indicate if changes 
were made. The images or other third party material in this article are included in the article's Creative Commons licence, unless indicated otherwise in a credit line to the material. If material is not included in the article's Creative Commons licence and your intended use is not permitted by statutory regulation or exceeds the permitted use, you will need to obtain permission directly from the copyright holder. To view a copy of this licence, visit http://creativecommons.org/licenses/by/4.0/.

\section{References}

1. Chang BS, Lowenstein DH (2003) Epilepsy. N Engl J Med 349(13):1257-1266

2. Becker AJ, Chen J, Paus S, Normann S, Beck H, Elger CE et al (2002) Transcriptional profiling in human epilepsy: expression array and single cell real-time qRT-PCR analysis reveal distinct cellular gene regulation. NeuroReport 13(10):1327-1333

3. McClelland S, Flynn C, Dubé C, Richichi C, Zha Q, Ghestem A et al (2011) Neuron-restrictive silencer factor-mediated hyperpolarization-activated cyclic nucleotide gated channelopathy in experimental temporal lobe epilepsy. Ann Neurol 70(3):454-465

4. Schickel R, Boyerinas B, Park S, Peter M (2008) MicroRNAs: key players in the immune system, differentiation, tumorigenesis and cell death. Oncogene 27(45):5959-5974

5. Wang J, Zhao J (2021) MicroRNA dysregulation in epilepsy: from pathogenetic involvement to diagnostic biomarker and therapeutic agent development. Front Mol Neurosci 14:35

6. Wang L, Song L, Chen X, Suo J, Ma Y, Shi J et al (2020) microRNA-139-5p confers sensitivity to antiepileptic drugs in refractory epilepsy by inhibition of MRP1. CNS Neurosci Ther 26(4):465-474

7. Fu M, Tao J, Wang D, Zhang Z, Wang X, Ji Y et al (2020) Downregulation of MicroRNA-34c-5p facilitated neuroinflammation in drug-resistant epilepsy. Brain Res 1749:147130

8. Li Y, Huang C, Feng P, Jiang Y, Wang W, Zhou D et al (2016) Aberrant expression of miR-153 is associated with overexpression of hypoxia-inducible factor- $1 \alpha$ in refractory epilepsy. Sci Rep 6(1):1-10

9. Li N, Pan J, Liu W, Li Y, Li F, Liu M (2020) MicroRNA-15a-5p serves as a potential biomarker and regulates the viability and apoptosis of hippocampus neuron in children with temporal lobe epilepsy. Diagn Pathol 15(1):1-7

10. Zhang X, Li X, Li B, Sun C, Zhang P (2020) miR-21-5p protects hippocampal neurons of epileptic rats via inhibiting STAT3 expression. Adv Clin Exp Med 29(7):793-801

11. Li R, Wen Y, Wu B, He M, Zhang P, Zhang Q et al (2020) MicroRNA-25-3p suppresses epileptiform discharges through inhibiting oxidative stress and apoptosis via targeting OXSR1 in neurons. Biochem Biophys Res Commun 523(4):859-866

12. Qi Y, Qian R, Jia L, Fei X, Zhang D, Zhang Y et al (2020) Overexpressed microRNA-494 represses RIPK1 to attenuate hippocampal neuron injury in epilepsy rats by inactivating the NF- $\mathrm{KB}$ signaling pathway. Cell Cycle 19(11):1298-1313

13. Zhao C, Yang F, Wei X, Zhang J (2021) miR-139-5p upregulation alleviated spontaneous recurrent epileptiform discharge-induced oxidative stress and apoptosis in rat hippocampal neurons via regulating the Notch pathway. Cell Biol Int 45(2):463-476

14. Geng J, Zhao H, Liu X, Geng J, Gao Y, He B (2021) MiR-101a-3p attenuated pilocarpine-induced epilepsy by downregulating c-FOS. Neurochem Res 46:1119-1128

15. Liu X, Liao Y, Wang X, Zou D, Luo C, Jian C et al (2017) MicroRNA expression profiles in chronic epilepsy rats and neuroprotection from seizures by targeting miR-344a. Neuropsychiatr Dis Treat 13:2037
16. Wu DM, Zhang YT, Lu J, Zheng YL (2018) Effects of microRNA-129 and its target gene c-Fos on proliferation and apoptosis of hippocampal neurons in rats with epilepsy via the MAPK signaling pathway. J Cell Physiol 233(9):6632-6643

17. Xie Y, Shao Y, Deng X, Wang M, Chen Y (2018) MicroRNA-298 reverses multidrug resistance to antiepileptic drugs by suppressing MDR1/P-gp expression in vitro. Front Neurosci 12:602

18. Alsharafi WA, Xiao B, Abuhamed MM, Bi F-F, Luo Z-H (2015) Correlation between IL-10 and microRNA-187 expression in epileptic rat hippocampus and patients with temporal lobe epilepsy. Front Cell Neurosci 9:466

19. Jimenez-Mateos EM, Arribas-Blazquez M, Sanz-Rodriguez A, Concannon C, Olivos-Ore LA, Reschke CR et al (2015) microRNA targeting of the P2X7 purinoceptor opposes a contralateral epileptogenic focus in the hippocampus. Sci Rep 5(1):1-17

20. Xiang L, Ren Y, Li X, Zhao W, Song Y (2016) MicroRNA-204 suppresses epileptiform discharges through regulating TrkBERK1/2-CREB signaling in cultured hippocampal neurons. Brain Res 1639:99-107

21. Ge B, Liu H, Liang Q, Shang L, Wang T, Ge S (2019) Oxytocin facilitates the proliferation, migration and osteogenic differentiation of human periodontal stem cells in vitro. Arch Oral Biol 99:126-133

22. Wang Q, Fan D, Xia Y, Ye Q, Xi X, Zhang G et al (2021) The latest information on the RIPK1 post-translational modifications and functions. Biomed Pharmacother 142:112082

23. Zheng P, Bin H, Chen W (2019) Inhibition of microRNA-103a inhibits the activation of astrocytes in hippocampus tissues and improves the pathological injury of neurons of epilepsy rats by regulating BDNF. Cancer Cell Int 19(1):1-14

24. Lu J, Zhou N, Yang P, Deng L, Liu G (2019) MicroRNA-27a-3p downregulation inhibits inflammatory response and hippocampal neuronal cell apoptosis by upregulating mitogen-activated protein kinase 4 (MAP2K4) expression in epilepsy: in vivo and in vitro studies. Med Sci Monit 25:8499

25. Yuan J, Huang H, Zhou X, Liu X, Ou S, Xu T et al (2016) MicroRNA-132 interact with $250 \mathrm{GAP} / \mathrm{Cdc} 42$ pathway in the hippocampal neuronal culture model of acquired epilepsy and associated with epileptogenesis process. Neural Plast 2016:1-14

26. Korotkov A, Broekaart DW, Banchaewa L, Pustjens B, van Scheppingen J, Anink JJ et al (2020) microRNA-132 is overexpressed in glia in temporal lobe epilepsy and reduces the expression of pro-epileptogenic factors in human cultured astrocytes. Glia 68(1):60-75

27. Xiang L, Ren Y, Cai H, Zhao W, Song Y (2015) MicroRNA-132 aggravates epileptiform discharges via suppression of BDNF/ TrkB signaling in cultured hippocampal neurons. Brain Res 1622:484-495

28. Huang H, Cui G, Tang H, Kong L, Wang X, Cui C et al (2019) Silencing of microRNA-146a alleviates the neural damage in temporal lobe epilepsy by down-regulating Notch-1. Mol Brain 12(1):1-12

29. Kong H, Wang H, Zhuo Z, Li Z, Tian P, Wu J et al (2020) Inhibition of miR-181a-5p reduces astrocyte and microglia activation and oxidative stress by activating SIRT1 in immature rats with epilepsy. Lab Invest 100(9):1223-1237

30. Ren L, Zhu R, Li X (2016) Silencing miR-181a produces neuroprotection against hippocampus neuron cell apoptosis post-status epilepticus in a rat model and in children with temporal lobe epilepsy. Genet Mol Res 15(1):1-11

31. Tang C, Gu Y, Wang H, Wu H, Wang Y, Meng Y et al (2018) Targeting of microRNA-21-5p protects against seizure damage in a kainic acid-induced status epilepticus model via PTEN-mTOR. Epilepsy Res 144:34-42

32. He F, Liu B, Meng Q, Sun Y, Wang W, Wang C (2016) Modulation of miR-146a/complement factor H-mediated inflammatory 
responses in a rat model of temporal lobe epilepsy. Biosci Rep 36:6

33. Fu H, Cheng Y, Luo H, Rong Z, Li Y, Lu P et al (2019) Silencing microRNA-155 attenuates kainic acid-induced seizure by inhibiting microglia activation. NeuroImmuno Modul 26(2):67-76

34. Korotkov A, Broekaart DW, van Scheppingen J, Anink JJ, Baayen JC, Idema $S$ et al (2018) Increased expression of matrix metalloproteinase 3 can be attenuated by inhibition of microRNA-155 in cultured human astrocytes. J Neuroinflamm 15(1):1-14

35. Huang L-G, Zou J, Lu Q-C (2018) Silencing rno-miR-155-5p in rat temporal lobe epilepsy model reduces pathophysiological features and cell apoptosis by activating Sestrin-3. Brain Res 1689:109-122

36. Cai Z, Li S, Li S, Song F, Zhang Z, Qi G et al (2016) Antagonist targeting microRNA-155 protects against lithium-pilocarpineinduced status epilepticus in C57BL/6 mice by activating brainderived neurotrophic factor. Front Pharmacol 7:129

37. Wu X, Wang Y, Sun Z, Ren S, Yang W, Deng Y et al (2019) Molecular expression and functional analysis of genes in children with temporal lobe epilepsy. J Integr Neurosci 18(1):71-77

38. Wang Y, Yang Z, Zhang K, Wan Y, Zhou Y, Yang Z (2021) miR$135 \mathrm{a}-5 \mathrm{p}$ inhibitor protects glial cells against apoptosis via targeting SIRT1 in epilepsy. Exp Ther Med 21(5):1-8

39. Chen DZ, Wang WW, Chen YL, Yang XF, Zhao M, Yang YY (2019) miR-128 is upregulated in epilepsy and promotes apoptosis through the SIRT1 cascade. Int J Mol Med 44(2):694-704

40. Gao X, Guo M, Meng D, Sun F, Guan L, Cui Y et al (2019) Silencing microRNA-134 alleviates hippocampal damage and occurrence of spontaneous seizures after intraventricular kainic acid-induced status epilepticus in rats. Front Cell Neurosci 13:145

41. Rodriguez AS, Engel T, Palfi A, Farrar GJ, Henshall DC, JimenezMateos EM (2017) Tubby-like protein 1 (Tulp1) is a target of microRNA-134 and is down-regulated in experimental epilepsy. Int J Physiol Pathophysiol Pharmacol 9(6):178

42. Sun J, Gao X, Meng D, Xu Y, Wang X, Gu X et al (2017) Antagomirs targeting microRNA-134 increase Limk1 levels after experimental seizures in vitro and in vivo. Cell Physiol Biochem 43(2):636-643

43. Jimenez-Mateos EM, Engel T, Merino-Serrais P, McKiernan RC, Tanaka K, Mouri G et al (2012) Silencing microRNA-134 produces neuroprotective and prolonged seizure-suppressive effects. Nat Med 18(7):1087-1094

44. Xiao D, Lv J, Zheng Z, Liu Y, Zhang Y, Luo C et al (2021) Mechanisms of microRNA-142 in mitochondrial autophagy and hippocampal damage in a rat model of epilepsy. Int J Mol Med 47(6): $1-12$

45. Wang J, Zheng Y, Cheng X, Xu F, Zhang P, Zhou X et al (2019) Inhibition of microRNA-34a suppresses epileptiform discharges through regulating notch signaling and apoptosis in cultured hippocampal neurons. Neurochem Res 44(5):1252-1261

46. Zhang S, Yu N, Arce RM (2020) Periodontal inflammation: Integrating genes and dysbiosis. Periodontol 82(1):129-142

47. Feng Y, Duan C, Luo Z, Xiao W, Tian F (2020) Silencing miR$20 a-5 p$ inhibits axonal growth and neuronal branching and prevents epileptogenesis through RGMa-RhoA-mediated synaptic plasticity. J Cell Mol Med 24(18):10573-10588

48. Feng X, Xiong W, Yuan M, Zhan J, Zhu X, Wei Z et al (2019) Down-regulated microRNA-183 mediates the Jak/Stat signaling pathway to attenuate hippocampal neuron injury in epilepsy rats by targeting Foxp1. Cell Cycle 18(22):3206-3222

49. Du Y, Chi X, An W (2019) Downregulation of microRNA$200 c-3 p$ reduces damage of hippocampal neurons in epileptic rats by upregulating expression of RECK and inactivating the AKT signaling pathway. Chem Biol Interact 307:223-233

50. Zhao Y, Lu C, Wang H, Lin Q, Cai L, Meng F et al (2020) Identification of hsa-miR-1275 as a novel biomarker targeting MECP2 for human epilepsy of unknown etiology. Mol Ther Methods Clin Dev 19:398-410

51. Zhu X, Yao Y, Liu Y, Zhou R, Zhang W, Hu Q et al (2019) Regulation of ADAM10 by microRNA-23a contributes to epileptogenesis in pilocarpine-induced Status Epilepticus mice. Front Cell Neurosci 13:180

52. Duan W, Chen Y, Wang XR (2018) MicroRNA-155 contributes to the occurrence of epilepsy through the PI3K/Akt/mTOR signaling pathway. Int J Mol Med 42(3):1577-1584

53. Rajman M, Metge F, Fiore R, Khudayberdiev S, Aksoy-Aksel A, Bicker $\mathrm{S}$ et al (2017) A microRNA-129-5p/Rbfox crosstalk coordinates homeostatic downscaling of excitatory synapses. EMBO J 36(12):1770-1787

54. Lee S-T, Jeon D, Chu K, Jung K-H, Moon J, Sunwoo J et al (2017) Inhibition of miR-203 reduces spontaneous recurrent seizures in mice. Mol Neurobiol 54(5):3300-3308

55. Wang D, Li Z, Zhang Y, Wang G, Wei M, Hu Y et al (2016) Targeting of micro RNA-199a-5p protects against pilocarpineinduced status epilepticus and seizure damage via SIRT 1-p53 cascade. Epilepsia 57(5):706-716

56. Arai MA, Akamine R, Tsuchiya A, Yoneyama T, Koyano T, Kowithayakorn $\mathrm{T}$ et al (2018) The Notch inhibitor cowanin accelerates nicastrin degradation. Sci Rep 8(1):1-10

57. Avansini SH, de Sousa Lima BP, Secolin R, Santos ML, Coan AC, Vieira AS et al (2017) MicroRNA hsa-miR-134 is a circulating biomarker for mesial temporal lobe epilepsy. PLoS ONE 12(4): 0173060

58. Ioriatti ES, Cirino MLA, Neto FSL, Velasco TR, Sakamoto AC, Freitas-Lima $\mathrm{P}$ et al (2020) Expression of circulating microRNAs as predictors of diagnosis and surgical outcome in patients with mesial temporal lobe epilepsy with hippocampal sclerosis. Epilepsy Res 166:106373

59. Leontariti M, Avgeris M, Katsarou MS, Drakoulis N, Siatouni A, Verentzioti A et al (2020) Circulating miR-146a and miR-134 in predicting drug-resistant epilepsy in patients with focal impaired awareness seizures. Epilepsia 61(5):959-970

60. Shen C-H, Zhang Y-X, Zheng Y, Yang F, Hu Y, Xu S et al (2019) Expression of plasma microRNA-145-5p and its correlation with clinical features in patients with refractory epilepsy. Epilepsy Res $154: 21-25$

61. Martins-Ferreira R, Chaves J, Carvalho C, Bettencourt A, Chorão R, Freitas J et al (2020) Circulating microRNAs as potential biomarkers for genetic generalized epilepsies: a three microRNA panel. Eur J Neurol 27(4):660-666

62. De Benedittis S, Fortunato F, Cava C, Gallivanone F, Iaccino E, Caligiuri ME et al (2021) Circulating microRNA: the potential novel diagnostic biomarkers to predict drug resistance in temporal lobe epilepsy, a pilot study. Int J Mol Sci 22(2):702

63. Raoof R, Jimenez-Mateos EM, Bauer S, Tackenberg B, Rosenow F, Lang J et al (2017) Cerebrospinal fluid microRNAs are potential biomarkers of temporal lobe epilepsy and status epilepticus. Sci Rep 7(1):1-17

64. Yan S, Zhang H, Xie W, Meng F, Zhang K, Jiang Y et al (2017) Altered microRNA profiles in plasma exosomes from mesial temporal lobe epilepsy with hippocampal sclerosis. Oncotarget $8(3): 4136$

65. Sun Y, Wang X, Wang Z, Zhang Y, Che N, Luo X et al (2016) Expression of microRNA-129-2-3p and microRNA-935 in plasma and brain tissue of human refractory epilepsy. Epilepsy Res 127:276-283

66. Wang J, Yu J-T, Tan L, Tian Y, Ma J, Tan C-C et al (2015) Genome-wide circulating microRNA expression profiling indicates biomarkers for epilepsy. Sci Rep 5(1):1-9

67. Boschiero MN, Camporeze B, Dos Santos JS, da Costa LB, Bonafé GA, de Souza Queiroz L et al (2020) The single nucleotide 
variant $\mathrm{n} 60 \mathrm{G}>\mathrm{C}$ in the microRNA-146a associated with susceptibility to drug-resistant epilepsy. Epilepsy Res 162:106305

68. Cui L, Tao H, Wang Y, Liu Z, Xu Z, Zhou H et al (2015) A functional polymorphism of the microRNA-146a gene is associated with susceptibility to drug-resistant epilepsy and seizures frequency. Seizure 27:60-65

69. Manna I, Labate A, Mumoli L, Pantusa M, Ferlazzo E, Aguglia U et al (2013) Relationship between genetic variant in pre-microRNA-146a and genetic predisposition to temporal lobe epilepsy: a case-control study. Gene 516(1):181-183
70. Li Y, Wang J, Jiang C, Zheng G, Lu X, Guo H (2016) Association of the genetic polymorphisms in pre-microRNAs with risk of childhood epilepsy in a Chinese population. Seizure 40:21-26

Publisher's Note Springer Nature remains neutral with regard to jurisdictional claims in published maps and institutional affiliations. 